\title{
Incidência de Lesões nos Jogadores de Futebol Masculino Sub-21 Durante os Jogos Regionais de Sertãozinho-SP de 2006
}

\section{Injury Incidence in Sub-21 Male Soccer Players During Regional \\ Games of Sertãozinho-SP 2006}

Luiz Fernando Approbato Selistre Oswaldo Luiz Stamato Taube ${ }^{2,3}$ Luciano Maia Alves Ferreira²

Edson Alves Barros Jr²

1. Universidade Federal de São Carlos-SP (UFSCar).

2. Centro Universitário Claretiano de Batatais-SP (Cecular).

3. Faculdades Integradas de Bebedouro-SP (Fafibe).

Endereço para correspondência: Luiz Fernando Approbato Selistre Rua Espírito Santo, 249,

Sumarezinho

14055-030 - Ribeirão Preto,SP

E-mail: Ifselistre@yahoo.com.br

Submetido em: 01/12/2008

Versão final recebida em: 30/04/2009 Aceito em: 11/05/2009

\section{RESUMO}

Introdução: O futebol, o esporte mais popular e praticado pelo mundo, é também uma das modalidades esportivas onde o atleta é submetido a diversos tipos e intensidades de esforços. Atualmente essa modalidade sofreu modificações, deixando de dar ênfase à técnica e passando a privilegiar os componentes físicos, aumentando assim a probabilidade da ocorrência de lesões. Objetivo: Realizar um levantamento epidemiológico das lesões no Futebol de Campo Sub-21 durante os 50s Jogos Regionais de Sertãozinho de 2006. Metodologia: Este é um trabalho do tipo epidemiológico descritivo e analítico, com desenho transversal. Realizou-se o acompanhamento de todas as partidas da modalidade, registrando as lesões ocorridas, seguido de um levantamento e caracterização das lesões. As mesmas foram classificadas de acordo com seu perfil e segmento anatômico, posteriormente divididas por posição dos atletas. Resultados: Houve maior incidência das lesões nos membros inferiores, com 127 (74,7\%); quanto ao perfil, as lesões musculares foram mais prevalentes, com $64(37,6 \%)$ lesões. Os atletas do meiocampo foram os mais acometidos, com $72(42,4 \%)$ lesões. Dentro da classe de goleiros e defensores a maior prevalência de contusões foi de sete $(63,6 \%)$ e 11 (50\%), respectivamente. Já os atacantes, laterais e meio-campistas apresentaram como predomínio as lesões musculares, sendo os primeiros com 11 $(36,7 \%)$, seguidos de $12(34,3 \%)$ dos laterais e $33(45,8 \%)$ dos meio-campistas. Conclusão: Constatou-se que ocorreu maior índice de lesões em membros inferiores, sendo estas caracterizadas como as musculares de maior prevalência. Demonstrou-se ainda que as lesões apresentadas estão diretamente relacionadas com as características de cada posição.

Palavras-chave: atleta, epidemiologia, membros inferiores.

\section{ABSTRACT}

Soccer is one of the most popular and practiced sports in the world. It is a sport in which athletes are submitted to many types and effort intensities. This sport has changed over the years, and instead of emphasizing technique, currently the physical components are more favored, increasing hence the injury risk. OBJECTIVE: The aim of this study was to perform an epidemiological survey of sub-21 male soccer player's injuries in the Regional Games 2006. METHODS: This study was of epidemiological descriptive and analytical type, with a transversal design. All games of the modality were followed, and the injuries found were registered by their prevalence and classification. Injuries were classified according to their profile and anatomical segment and according to athletes' position as well. RESULTS: The highest prevalence of injuries was found in the lower extremities 127 (74.7\%), and muscular injuries were the most frequent 64 (37.6\%). Midfielders were the most affected players 72 (42.4\%). The most frequent injury type in goalkeepers and defenders were contusions with 7 (63.6\%) and 11 (50\%), respectively. Strikers, outside midfielders and midfielders showed mostly muscular injuries, with 11 (36.7\%), 12 (34.3\%) and 33 (45.8\%), respectively. CONCLUSIONS: In conclusion, this study demonstrated higher incidence of injuries in lower extremities in sub-21 soccer players, being muscular injuries the most common ones. The results also suggest that injuries are directly related with the characteristics of each athlete's position.

Keywords: athlete, epidemiology, lower extremities. 


\section{INTRODUÇÃO}

O futebol é o esporte mais popular do mundo, difundido na maioria dos países, especialmente latino-americanos e europeus, contando com 208 países associados à Federação Internacional de Futebol e cerca de 200 milhões de praticantes. A origem mais conhecida é a atribuída ao inglês Charles Miller, que trouxe a modalidade da Inglaterra para o Brasil, em 1874. A FIFA (Fédération Internationale de Football Association) é responsável pela organização do esporte, determinando as regras, calendário de eventos mundiais e alterações relacionadas ao jogo ${ }^{(1,2)}$.

Atualmente, o mundo esportivo é caracterizado pela forte tendência ao profissionalismo, trazendo consigo implícitas as variáveis de intensidade, frequência e duração, aumentadas de forma não coerente com as condições dos atletas ${ }^{(3,4)}$. Esses fatores acabam ocasionando níveis importantes de estresse físico e mental, influenciando na saúde, qualidade de vida e futuro desses atletas ${ }^{(5)}$. É consenso internacional que houve modificação nas características do futebol, com a substituição do futebol-arte pelo futebol-força ${ }^{(6,7)}$.

Alguns fatores são variantes importantes que, quando irregulares, predispõem o atleta a lesões; podem ser intrínsecos (idade, sexo, capacidade física) ou extrínsecos (material utilizado, treinamento, locais de jogos) ${ }^{(8-11)}$. Diante dessa realidade, a fisioterapia vem se mostrando a cada vez mais presente nesse esporte, atuando na reabilitação e/ou prevenção(12-16). As lesões encontradas no futebol podem ser classificadas como: contusão, lesão muscular, fratura, tendinite, entorse, subluxação e luxação(1,17-19).

Os Jogos Regionais tiveram início em 1950, na cidade de Presidente Prudente-SP, com o objetivo de promover a prática e o intercâmbio esportivo. O evento é o segundo mais importante dentro do Estado de São Paulo, superado apenas pelos Jogos Abertos do Interior. Os Jogos Regionais das diferentes regiões são classificatórios para os Jogos Abertos do Interior, a maior competição da América Latina.

O objetivo deste trabalho foi realizar um levantamento epidemiológico das lesões no futebol de campo masculino Sub-21 durante os $50^{\circ} \mathrm{S} J$ Jogos Regionais realizados na cidade de Sertãozinho-SP no ano de 2006. Pretendeu-se demonstrar de forma quantitativa as lesões encontradas na modalidade, relacionada com as posições dos jogadores.

\section{METODOLOGIA}

Este estudo é do tipo epidemiológico analítico, com desenho transversal e foi aprovado pelo Comitê de Ética em Pesquisa das Universidades Integradas de Bebedouro (Fafibe) (Parecer nº 0091/2008), de acordo com as normas da Resolução 196/96 do Conselho Nacional de Saúde sobre pesquisa envolvendo seres humanos.

Foram acompanhadas 51 equipes, compostas por 22 jogadores, totalizando 1.122 atletas, com idade entre 16 e 20 anos (18 \pm 2 ). 0 acompanhamento ocorreu durante as 52 partidas realizadas, com duração de 70 minutos cada, entre os dias 5 e 11 de julho de 2006 nos períodos da manhã e tarde, por graduandos do Projeto de Fisioterapia Desportiva do Centro Universitário Claretiano de Batatais (Ceuclar), além de graduandos do curso de Enfermagem da mesma instituição, supervisionados por docentes dos cursos. Os estudantes permaneciam nas partidas e, quando necessário, adentravam no campo e realizavam o atendimento do atleta, registrando os dados em uma ficha, a qual continha: nome, data de nascimento, posição, equipe e lesão. As lesões foram registradas de acordo com o consenso descrito por Fuller et al. ${ }^{(20)}$, que define lesão como sendo apenas os acometimentos que ocorrem durante as partidas e que causam ao jogador uma perda de jogo, ou seja, o mesmo deveria abandonar a partida. As lesões relatadas pelos atletas, causadas por eventos antigos, de partidas, treinos da modalidade ou outras atividades, não foram consideradas para registro.
As posições consideradas foram: goleiro, defesa, lateral, meio-campo e atacante. Os tipos de lesões foram classificados como: contusão, lesão muscular (estiramento muscular, contusão muscular, câimbra, dolorimento muscular tardio), entorse, tendinite, fratura e luxação(17,19,21). Foram classificadas ainda quanto ao segmento: membros superiores (MMSS), membros inferiores (MMII), tronco e cabeça/pescoço (20). A análise dos dados permitiu a obtenção do número de lesões por partida, quantidade de lesões por 1.000 partidas disputadas e quantidade de lesões por 1.000 horas de jogo, além de quantidade de lesões pelo tipo e segmento acometido, posição mais acometida, tipo de lesão por posição e segmento acometido por posição. Os cálculos foram feitos com base nas fórmulas de utilizadas por Junge et al. ${ }^{(17)}$.

Ao final do evento, os formulários utilizados para registro dos dados foram arquivados e posteriormente analisados para a realização do estudo. Os dados foram colocados em forma de gráficos no programa Excel' (Microsoft Office 2007), para melhor demonstração dos resultados obtidos e realizados os intervalos de confiança.

\section{RESULTADOS}

A partir da análise dos prontuários, foram registradas 170 lesões. Obteve-se que ocorreram em média 3,3 lesões por partida (95\% IC 2,83,8), 148,6 lesões por 1.000 partidas disputadas (95\% IC 126,3-170,9) e 128,1 lesões por 1.000 horas de jogo (95\% IC 108,8-147,4). A quantidade de lesões por tipo foi de: 64 (37,6\%) lesões musculares, 52 (30,6\%) contusões, 40 (23,5\%) entorses, sete $(4,1 \%)$ tendinites, cinco $(2,9 \%)$ luxações e duas (1,2\%) fraturas (figura 1). A quantidade de lesões por segmento foi de: 14 (8,2\%) lesões em MMSS, 127 (74,7\%) em MMII, 19 (11,2\%) em tronco e 10 (5,9\%) em cabeça e pescoço.

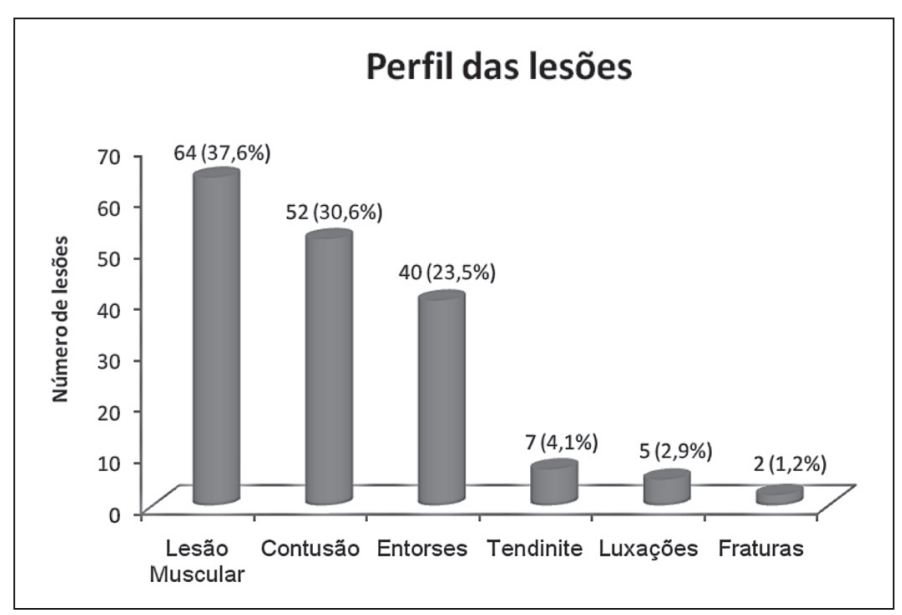

Figura 1. Perfil das lesões encontradas em jogadores de futebol masculino sub-21 durante os Jogos Regionais de 2006. Resultados expressos em números absolutos e porcentagem.

As posições mais acometidas por lesão foram: meio-campo com 72 (42,4\%) lesões, seguida por lateral 35 (20,6\%), atacante 30 (17,6\%), defesa 22 (12,9\%) e goleiro 11 (6,5\%) lesões.

A análise dos tipos de lesão por posição dos jogadores mostrou: goleiros com uma $(9,1 \%)$ lesão muscular, sete $(63,6 \%)$ contusões, duas $(18,2 \%)$ entorses e uma $(9,1 \%)$ luxação. Nos jogadores de defesa foram encontradas sete $(33,1 \%)$ lesões musculares, 11 (39,3\%) contusões articulares e quatro $(23,4 \%)$ entorses. Nos laterais foram 12 (34,3\%) lesões musculares, 10 (28,6\%) contusões articulares, 10 (28,6\%) entorses, duas $(5,7 \%)$ tendinites e uma $(2,8)$ luxação. Nos meias foram $33(45,8 \%)$ lesões musculares, 15 (20,8\%) contusões, 18 (25\%) entorses, quatro (5,6\%) tendinites e duas (2,8\%) luxações. Nos atacantes foram 11 (36,7\%) lesões musculares, nove (30\%) contusões, seis (20\%) entorses, uma (3,3\%) tendinite, uma $(3,3 \%)$ luxação e duas $(6,7 \%)$ fraturas (figura 2$)$. 


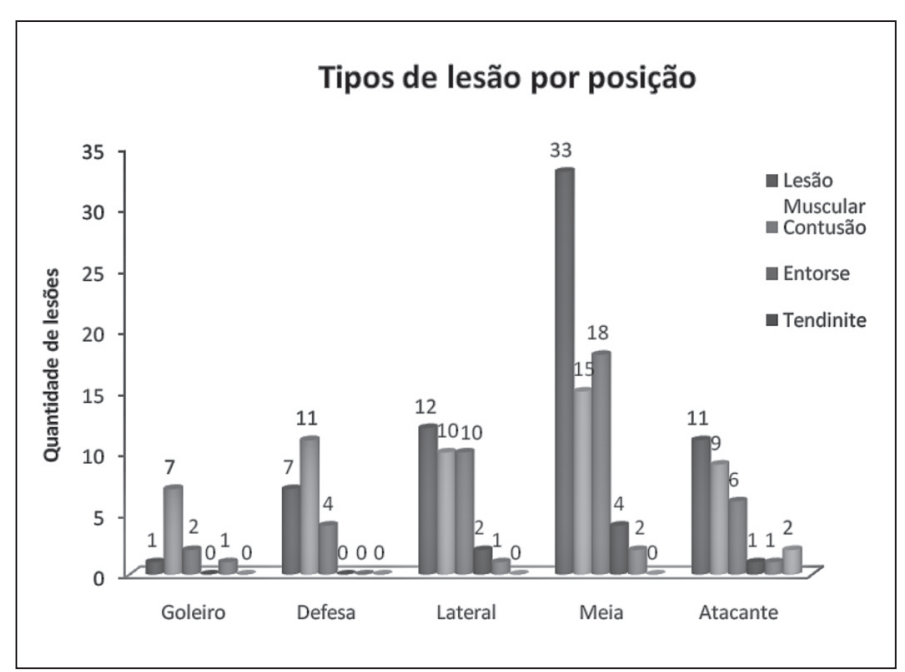

Figura 2. Tipos de lesões encontradas por posição em jogadores do futebol masculino sub-21 durante os Jogos Regionais de 2006. Resultados expressos em números absolutos.

Os segmentos acometidos por posição dos jogadores apresentaram: goleiros com três $(27,3 \%)$ lesões em MMSS, cinco $(45,4 \%)$ em MMII, uma (9,1\%) em tronco e duas (18,2\%) em cabeça/pescoço; os defensores apresentaram uma (4,5\%) lesão em MMSS, 13 (59,1\%) em MMII, quatro (18,2\%) de tronco e quatro (18,2\%) em cabeça/pescoço; em laterais foram cinco (14,3\%) em MMSS, 22 (62,9\%) em MMII e sete (20\%) em tronco e uma $(2,9 \%)$ em cabeça/pescoço; nos meias foram três (4,2\%) lesões em MMSS, 60 (83,3\%) em MMII, seis (8,3\%) em tronco e três (4,2\%) em cabeça/pescoço; atacantes apresentaram duas (6,7\%) lesões em MMSS, 27 (90\%) em MMII e uma (3,3\%) em tronco (figura 3).

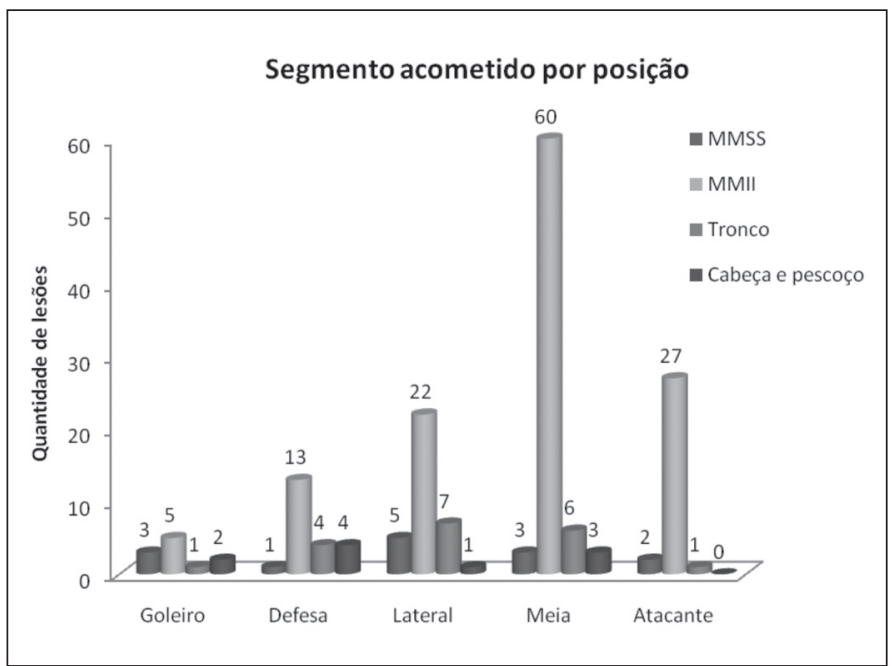

Figura 3. Segmentos acometidos por posição dos jogadores de futebol masculino sub21 durante os Jogos Regionais de 2006. Resultados expressos em números absolutos.

\section{DISCUSSÃO}

Atualmente, a literatura apresenta vários estudos que abordam a epidemiologia de lesões no futebol(22-26). Esses estudos variam entre si quanto às metodologias utilizadas, além de abordar diferentes amostras. Apesar disso, o registro das lesões parece ser o ponto fundamental das pesquisas epidemiológicas, pois permitem a comparação com outros trabalhos realizados ou contribuem para trabalhos futuros ${ }^{(27)}$.

O presente estudo apontou média de 3,3 lesões por jogo, 128,1 lesões por 1.000 horas de jogo e 148,6 lesões por 1.000 partidas dispu- tadas. Já no estudo de Junge et al. ${ }^{(17)}$, em 32 partidas, foram registradas 77 lesões, obtendo-se média de 2,4 lesões por partida e 108 por 1.000 partidas disputadas. Em outro estudo, Junge et al. ${ }^{(6)}$ acompanharam 64 partidas, registraram 171 lesões, encontrando média de 2,7 lesões por partida ou 81,0 lesões por 1.000 horas de jogo.

O tipo de lesão mais encontrado foi de lesões musculares (37,6\%); resultados contrários foram encontrados por Junge et al. ${ }^{(17)}$, que encontraram maior quantidade de contusões (70\%), seguidas pelo mesmo número de lesões musculares e entorses (10\%), dados colhidos durante os Jogos Olímpicos de 2004. Em outro estudo(19), foi realizado o acompanhamento de jogos universitários dos Estados Unidos no período de 2005 a 2007; os autores encontraram maior quantidade de entorses (26,8\%), seguidos por lesões musculares $(17,9 \%)$ e contusões $(13,8 \%)$. Le Gall et al. ${ }^{(21)}$ encontraram maior incidência de contusões $(30,6 \%)$, seguidas por entorses (16,7\%), lesões musculares (15,3\%), fraturas (5,9\%) e luxação (0,9\%), análise essa realizada com jogadores profissionais da divisão principal do futebol francês durante dez temporadas. Segundo Junge et al. ${ }^{(6)}$, essa grande variedade quanto à incidência de lesões parece estar relacionada com o tipo de torneio e característica dos jogadores.

Quanto ao seguimento acometido, os membros inferiores demonstraram ser mais lesionados, seguidos por tronco, MMSS e cabeça/pescoço. Resultado semelhante foi encontrado por Rechel et al. ${ }^{(28)}$ que, de um total de 334 lesões, observaram maior acometimento de MMII $(65,1 \%)$, entretanto, seguidos por cabeça/pescoço $(19,7 \%)$, MMSS $(8,4 \%)$ e tronco (6,8\%). O estudo de Agel et al. ${ }^{(29)}$, também encontrou maior incidência em MMII (67,3\%), seguidos por cabeça/pescoço (12,8\%), tronco $(10,5 \%)$ e MMSS (6,8\%) de um total de 6.693 lesões registradas. Além desses, outros estudos ${ }^{(19,26,30)}$ apontam os MMII como mais lesionados, o que aparenta ser um consenso na modalidade em questão(1).

A dos meio-campistas demonstrou-se ser a posição mais acometida por lesão, seguida por laterais, atacantes, defensores e goleiros. Apesar da pequena quantidade de estudos que abordam os tipos de lesões por posição, existe o de Le Gall et al.(21), na qual os autores apontam a dos meio-campistas como a posição mais acometida por lesão (37,1\%), seguida por defensores (26,1\%), atacantes $(22,7 \%)$ e goleiros (14,1\%), de um total de 1.152 lesões registradas.

Este estudo apresenta limitações quanto ao registro das lesões, pois não foi observado o tempo de afastamento dos atletas, assim como a severidade dessas lesões, devido à forma como foram realizadas as coletas, além de não apontar os mecanismos de lesão, característica essa que se torna importante para uma futura intervenção preventiva das equipes participantes. Por outro lado, trata-se de um dos primeiros estudos para esse tipo de competição, o que traz consigo um incentivo para publicações futuras, além de contribuir para um conhecimento mais amplo e abrangente do perfil dos jogadores e torneios de futebol do país.

\section{CONCLUSÃO}

A partir dos resultados apresentados, conclui-se que as lesões musculares são mais incidentes, o que parece estar relacionado com a curta duração da competição; além disso, os jogadores de meio-campo apresentam maior quantidade de lesões. O segmento mais acometido foi o dos membros inferiores, assim como nos outros trabalhos encontrados. Os tipos de lesões parecem estar relacionados com as características do jogador e sua posição em campo.

\section{AGRADECIMENTOS}

Agradecemos a colaboração da Profa. Dra. Heloisa Sobreiro Selistre de Araújo e da Profa. Dra. Rita de Cássia Marqueti, da Universidade Federal de São Carlos-SP (UFSCar), pela atenção investida e leitura crítica do manuscrito. 


\section{REFERÊNCIAS BIBLIOGRÁFICAS}

1. Manning MR, Levy RS. Soccer. Phys Med Rehabil Clin N Am. 2006;17:677-95

2. Kofotolis ND, Kellis E, Vlachopoulos SP. Ankle Sprain Injuries and Risk Factors in Amateur Soccer Players During a 2-Year Period. Am J Sports Med. 2007;35:458-66.

3. Rahnama N, Reilly T, Lees A. Injury risk associated with playing actions during competitive soccer. Br J Sports Med. 2002;36:354-9.

4. McGuine, T. Sports Injuries in High School Athletes: A Review of Injury-Risk and Injury-Prevention Research. Clin J Sport Med. 2006;16:488-99.

5. Thelin N, Holmberg S, Thelin A. Knee injuries account for the sports-related increased risk of knee osteoarthritis. Scand J Med Sci Sports. 2006;16:329-33.

6. Junge A, Dvorak J, Graf-Baumann T. Football Injuries During the World Cup 2002. Am J Sports Med. 2004;32:23-7.

7. Hägglund $M$, Waldén M, Ekstrand J. Previous injury as a risk factor for injury in elite football: a prospective study over two consecutive seasons. Br J Sports Med. 2006;40:767-72

8. Junge A, Rösch D, Peterson L, Graf-Baumann T, Dvorak J. Prevention of Soccer Injuries: A Prospective Intervention Study in Youth Amateur Players. Am J Sports Med. 2002;30:652-9.

9. Witvrouw E, Danneels L, Asselman P, D'Have T, Cambier D. Muscle Flexibility as a Risk Factor for Developing Muscle Injuries in Male Professional Soccer Players: A Prospective Study. Am J Sports Med. 2003;31:41-6.

10. Parkkari J, Kannus P, Natri A, Lapinleimu I, Palvanen M, Heiskanen, et al. Int J Sports Med 2004; 25:209-16.

11. Arnason A, Andersen TE, Holme I, Engebretsen L, Bahr R. Prevention of Hamstring strains in elite soccer: an intervention study. Scand J Med Sci Sports. 2008;18:40-8.

12. Hägglund M, Waldén M, Ekstrand J. Lower Reinjury Rate With a Coach-Controlled Rehabilitation Program in Amateur Male Soccer. Am J Sports Med. 2007;35:1433-42.

13. Bradley PS, Portas MD. The Relationship Between Preseason Range of Motion and Muscle Strain Injury in Elite Soccer Players. J Strength Cond Res. 2007;21:1155-9.

14. Engebretsen AH, Myklebust G, Holme I, Engebretsen L, Bahr R. Prevention of Injuries Among Male Soccer Players: A Prospective, Randomized Intervention Study Targeting Players With Previous Injuries or Reduced Function. Am J Sports Med. 2008;36:1052-60.

15. Croisier JL, Ganteaume S, Binet J, Genty M, Ferret JM. Strength Imbalamces and Prevention of Hamstring Injury in Professional Soccer Players: A Prospective Study. Am J Sports Med. 2008;36:1469-75.
16. Greig M, Siegler JC. Soccer-Specific Fatigue and Eccentric Hamstrings Muscle Strength. J Athl Train. 2009;44:180-4.

17. Junge A, Langevoort G, Pipe A, Peytavin A, Wong F, Mountjoy M, et al. Injuries in Team Sport Tournaments During the 2004 Olympic Games. Am J Sports Med. 2006;34:565-76.

18. Heidt RS, Sweeterman LM, Carlonas RL, Traub JA, Tekulve FX. Avoidance of Soccer Injuries with Preseason Conditioning. Am J Sports Med. 2006;28:659-62.

19. Yard EE, Schroeder MJ, Fields SK, Collins CL, Comstock RD. The Epidemiology of United States High School Soccer Injuries, 2005 2007. Am J Sports Med. 2008;36:1930-7.

20. Fuller CW, Ekstrand J, Junge A, Andersen TE, Bahr R, Dvorak J, Hägglund M, et al. Consensus Statement on Injury Definitions and Data Collection Procedures in Studies of Football (Soccer) Injuries. Clin J Sports Med. 2006;16:97-106.

21. Le Gall F, Carling C, Reilly T, Vandewalle H, Church J, Rochcongar P. Incidence of Injuries in Elite French Youth Soccer Players: A 10-Season Study. Am J Sports Med. 2006;34:928-38.

22. Hägglund $M$, Waldén $M$, Ekstrand J. Injury incidence and distribution in elite football - a prospective study of the Danish and the Swedish top divisions. Scand J Med Sci Sports. 2005;15:21-8.

23. Waldén $M$, Hägglund M, Ekstrand J. Injuries in Swedish elite football - a prospective study on injury definitions, risk for injury and injury pattern during 2001. Scand J Med Sci Sports. 2005;15:118-25a.

24. Waldén M, Hägglund M, Ekstrand J. Football injuries during European Championships 2004 - 2005. Knee Surg Sports Traumatol Arthrosc. 2007;15:1155-62.

25. Huffman EA, Yard EE, Fields SK, Collins CL, Comstock RD. Epidemiology of Rare Injuries and Conditions Among United States High School Athletes During the 2005-2006 and 2006-2007 School Years. J Athl Train. 2008;43:624-30

26. Palacio EP, Candeloro BM, Lopes AA. Lesões nos Jogadores de Futebol Profissional do Marília Atlético Clube: Estudo de Coorte Histórico do Campeonato Brasileiro de 2003 a 2005. Rev Bras Med Esporte. 2009;15:31-5.

27. Ekstrand J. Epidemiology of football injuries. Science \& Sports. 2008;23:73-7.

28. Rechel JA, Yard EE, Comstock RD. An Epidemiologic Comparison of High School Sports Injuries Sustained in Pratice and Competition. J Athl Train. 2008;43:197-204.

29. Agel J, Evans TA, Dick R, Putukian M, Marshall SW. Descriptive Epidemiology of Collegiate Men's Soccer Injuries: National Collegiate Athletic Association Injury Surveillance System, 1988-1989 Through 20022003. J Athl Train. 2007;42:270-7.

30. Waldén M, Hägglund M, Ekstrand J. UEFA Champions League study: a prospective study of injuries in professional football during the 2001-2002 season. Br J Sports Med. 2005;39:542-6b. 